directed to the improvement of the general health; this was, in some degree, effected; her strength, however, failed; morning sweats and increased pain in the ham rendered it necessary to amputate the limb on the 17 th of September.

\section{Examination of the Joint.}

The cellular membrane covering the joint was very much thickened, and indurated, in some parts presenting a glandular appearance. The swelling commenced three inches above the condyles, and extended to their extremities; it was globular, and on cutting through the shaft of the femur longitudinally, it was found to be osteo-sarcoma. Several parts were softened, and contained small bony particles; in the ham, or rather between the condyles, where she complained of the pain, was a cavity containing about th ounce and half of coagulated blood; the skin covering this was discoloured; at the bottom of this cavity was the same description of bony particles as alluded to above ; the substance of the swelling was so hard, as to render it difficult to cut. The interior of the joint was healthy, with the exception of the internal ligaments, which were dark coloured and much thickened.

Since the operation the general health has much improved, and the stump is nearly healed.

\section{LIVERPOOL MEDICAL INSTITUTION.}

The first ordinary meeting of the members of the Liverpool Medical Institution was held on 'Thursday, the 1st of October, for the purpose of electing four members as vicepresidents, whose duties are to preside, in rotation, at the ordinary meetings of the institution. At these meetings papers are read, and medical intelligence received. There are upwards of 150 members of the institution, to which is attached a large and extensive library. Each member subscribes $2 l$. 2s. annually, and at least one-third of the amount must be expended in books; the remaining twothirds are appropriated to the incidental expenses of the building, which is very handsome, having a front of white stone; it was erected by public contributions, but principally by the munificence of the late Dr. Rutter. The gentlemen elected as vice-presidents were, Dr. Freckleton, Dr. James Vose, Dr. Duncan, and Mr. Banner. Dr. Sutherland was re-elected secretary.

\section{ST. MARYLEBONE INFIRMARY.}

CASES OF ABSCESSES OCCURRING IN THE BRAIN. BY R. BOY D, M.D. Resident Physician.

MARY S—, æ- at. 19, admitted in the last stage of phthisis, states that she has been subject to spitting of blood on making any exertion, for the last eleven years; has been getting gradually worse for the last three months; the spitting of blood during the last week amounted to about three pints. Her mother died under my care of phthisis about three years ago, at which period this girl had medicine prescribed for her, of which, or of her symptoms, no note was taken at the time. I am indebted to Dr. Harrison (whose patient she was in the Infirmary) for the following brief note of her symptoms on admission: pulse rather quick, jerking, compressible; skin hot; tongue whitish ; bowels open, though usually confined; catamenia ceased four months ago; severe head-ache during the last three days; nausea for the last week; vomited this morning some greenish coloured matter. On examining the chest, a dull sound was elicited by percussion, under the right clavicle,' with considerable flattening of that region, \&c. During the two following days the headache became pro-
gressively worse. Considerable excitement now existed, and she often shrieked out from the intensity of the pain. The vomiting still continued; pupils slightly dilated. Following day, more composed; pain less acute; seemed afraid to move her head from the pillow; spoke in an under tone. The next morning, little apparent change.
She could get out of bed without any assistance, when she had occasion to do so. About half an hour after this she died quite tranquilly.

\section{Autopsy 25 hours after Death.}

Head only allowed to be examined.-On removing the dura mater, a slight cohesion was found to exist betwixt the surfaces of the arachnoid, which presented a dull glazed appearance; it communicated to the touch a feeling of dryness, with unctuosity; the convolutions appeared flattened; veins considerably distended with blood. On the superior, posterior, and outer portion of the left hemisphere, was observed a depôt of purulent matter, as large as a shilling, extending into the substance of the brain. The anterior half of the body of the lateral ventricle was filled with pus, streaked with blood. On examination, a deposit about the same size as the first was found in the inferior and inner portion of the corpus striatum. A smaller deposit existed in the inferior, anterior, and outer portion of the left hemisphere ; and a fourth, still smaller, was found in the cortical substance of the upper and anterior portion of the right hemisphere. On the surface of the arachncid, at the junction of the pons Varolii with the medulla oblongata, there was a yellowish deposit, probably the result of inflammatory action. The interior of each of the cavities containing the purulent deposits, seemed to be lined with a distinct membrane; that lining the small cavity in the cortical substance was smoother than those of the others, which were all more or less irregular in their interior, especially that in the corpus striatum, on the inferior surface of which there were some enlarged vessels, and the pus in this situation was mixed with a little blood. The matter in all the cavities, on examination, presented the characters of healthy pus.

The symptoms of cerebral disturbance were only complained of eight days before death; and they did not arrive at their height till forty-eight hours before that event, when she presented all the symptoms of a person labouring under acute arachnitis. I recollect a male insane patient, under the care of the late Dr. Sims, exhibiting very similar symptoms, followed by death. He said at the time we should probably find a dryness of the arachnoid membrane. The post-mortem examination proved that his opinion was correct. The late Dr. Hooper, of this institution, has recorded a few cases of a similar nature. The works of Morgagni, Portal, Bailly, Abercrombie, \&c. furnish several examples. A case occurred in the practice of this infirmary, of abscess in the " pons Varolii," which was exhibited by Mr. Obré, at one of the early meetings of the Patholological Society last season. (Vide Lancet, and Medical Gazette, March 28th, 1840.) Such cases are, notwithstanding, very rare ; only two or three examples of it having occurred, out of many hundreds of examinations of cerebral complications made at this infirmary during the last six years. Cerebral symptoms at the termination of phthisis are by no means uncommon. From an analysis of fifteen cases of that disease, which were examined here during the autumn of 1839 , it appears, two cases were attended with delirium; in one of these there was softening of the septum lucidum, and about $3 \mathrm{oz}$. of fluid in the lateral ventricles. The other case also contained a somewhat smaller quantity of fluid in the lateral ventricles.

Dr. Clendinning, in one of his Clinical Lectures here last year, related the case of a young man, who was admitted into the infirmary in a state of delirium, and complaining by various intelligible gestures and cries, of intense pain of the head, without coma, or affection of the pupils, and making no other complaint, nor seeming to suffer at all otherwise. He was wasted, and had a slight cough, without expectoration. There was dulness on percussion, and depression of both subclavian regions. The restlessness of the patient prevented satisfactory auscultation; but the case needed no further light than that elicited by the fingers for decisive diagnosis. After ten days or a fortnight, he sank. The membranes of the brain, too, were congested, and pure serum, amounting to above $2 \mathrm{oz}$, existed in the ventricles. 
The convolutions moderately flattened. There was no pus, or lymph, or thickening, or other unequivocal evidence of inflammation in the head. Large excavations existed in the apices of the lungs. The closing scenes of other chronic diseases are also occasionally marked by delirious excitement, resembling that of arachnitis. It often happens in such cases, that we are unable to detect any alteration of structure, and have to rest satisfied with denominating it a functional derangement.

Oct. 1, 1840.

\section{FOREIGN MEDICAL LITERATURE.} Archives Gen. de MÉ decine, August, 1840.

The August number of this journal contains the following original articles :

1. On the respiratory sounds in a state of health and during disease, by M. Beau (article not finished).

2. Case of amputation of the superior maxillary bone, for the removal of a nasal polypus, by $M$. Flaubert.

3. Medico-legal remarks on a case of fracture of the skull, by M. Ollivier d'Angers.

4. On a case of complete luxation of the foot, backwards, by $\mathbf{M}$. Ballot.

5. Experiments on the functions of the pneumogastric nerve, and on the internal branch of the accessory, by Professor Arnold of Zurich.

The case of removal of the superior maxillary bone, recorded by $M$. Flaubert, differs from all those which have been performed by other surgeons, in the circumstance that the operation was undertaken, not on account of any disease of the bone, but in order to get access to a very large polypus, which occupied the nasal fossæ and back of the throat. After various attempts to remove the polypus by ligature, and after having ineffectually divided the hard palate and cut away large portions of the tumour, M. Flaubert determined on removing the superior maxillary bone. This was done in the usual manner, and the polypus finally separated from all its attachments, with the knife and with a curved pair of scissors. The inflammation which succeeded this very severe operation was comparatively trifling, and the patient was dismissed the hospital, cured, on the fifteenth day.

The observations of $M$. Ollivier d'Angers refer to a very remarkable case which occurred in the month of June, 1839. The fosse d'aisance (water-closet) of a house in the street St. Denis had been emptied, and the inspector, having examined it, had commanded that it should be closed up. A mason was sent for, but before his arrival, it appears that a young man named Duchesne entered the premises and fell headlong into the hole. The mason came in a few minutes afterwards, and closed the opening.

Three days and three nights passed over without the slightest noise or groan being heard; but, on the fourth day, some of the lodgers in the house fancied that they heard some stifled groans which seemed to issue from the earth. On the following night the porter heard the same cries more distinctly: he got up, and examined all the cellars, but could discover nothing. The groans were succeeded by profound silence, and the agony of the unfortunate sufferer had continued for eight days and eight nights, when his groans were again distinctly heard. The trench was now examined, and Duchesne was discovered immediately beneath the seat over the trench, with his back against the wall. He breathed, but was unable to speak, and died on his way to a neighbouring hospital. On examining the body, after death, amongst several other injuries, it was found that the skull was extensively fractured, and that three ounces of dark blood, quite dried up, had been effused underneath the fractured bones, depressing in a very marked manner the anterior and lateral lobes of the brain on the right side. The other organs of the body were dried up, and presented the appearances which are seen in persons who have died after long abstinence from food.

The most remarkable circumstance in the above case is the influence which the forced abstinence from all nourishment had on the absorption of blood within the skull. During the first four days the unfortunate young man must have lain in a state of profound coma and paralysis, from the effects of the large quantity of blood effused upon the brain; but, at the end of that time, absorption had gone on so rapidly, as to admit of partial recovery of the reason, and to allow him to drag himself several feet from the point at which he fell into the trench towards the opening at the seat, near which he was found.

At the meeting of the British Association, which took place at Newcastle, Dr. Reed communicated the results'of some interesting experiments which he had performed on the pneumogastric nerves. The principal result obtained by Dr. Reed was, that when the vagi and recurrent nerves were divided in dogs, " a great diminution took place in the number of the respiratory movements, which, at the same time, became slower and more heaving."

The experiments of $\mathrm{M}$. Arnold were performed on pigeons and hens. We must refer our readers to the "Archives" for details: the following are the conclusions at which the professor arrives. The pneumogastric is a purely sensitive nerve, and communicates impressions to the brain more or less vividly, according to the mucous membrane to which it is distributed. It communicates the sensations of hunger and the want of breathing; it has no direct influence on the secretion, the quantity, or the quality of the gastric juice, or on the contractions of the csophagus and stomach ; on the formation of chyme; on the muscles of the glottis ; on the respiration; on animal heat, or on the contractions of the heart. But when divided, it no longer communicates the want of breathing, and hence the number of respirations diminish, and various functions suffer from accumulation of blood about the chest. The internal branch of the spinal accessory presides over the contractions of the œsophagus, the muscles of the glottis, and the stomach.

\section{ON THE CONDITION OF THE BLOOD IN : VARIOUS DISEASES.}

\section{By MM. Andrat and Gayarret.}

M. Andral, it appears, has at length commenced preparing a way to the Institute. The following is an analysis of a valuable paper presented by him to that learned body, on the 27th of July last. M. Andral's memoir is founded on an examination of the blood in two hundred patients; the number of bleedings made was three hundred and sixty. In different diseases the proportion of fibrine varied from 1 to 10 in a thousand parts of blood; the globules from 21 to 185 ; the solid constituents of the serum from 57 to 104 ; and the water from 725 to 915 .

* It rarely happens in disease that the different principlea of the blood increase or diminish simultaneously. Occasionally, however, two of the constituent parts are modified at the same time, in an inverse proportion. Diseases, so far as regards the changes which they produce in the composition of the blood, may be divided into four classes. The first comprehends those in which the quantity of fibrine is constantly increased. Inflammations hold a chief rank in this class. The second comprises diseases in which the fibrine is never increased, but sometimes diminished; -fevers, for example. In the third class we constantly find a diminution of the blood-globules. Finally, in the fourth class the fundamental change consists in a diminution of the albumen of the serum, as in Bright's disease.

Facts, however, do not always present themselves to us 